\title{
Review on Performance Analysis of BER Using BPSK
}

\author{
Mrs. S.M. Patil ${ }^{1}$, Miss. Prachi Zodpe ${ }^{2}$, Miss. Shital Ragit ${ }^{3}$ \\ Assistant Prof., E\&TC, Government College of Engineering, Jalgoan, India ${ }^{1}$ \\ M.Tech, E\&TC, Government College of Engineering, Jalgoan, India ${ }^{2,3}$
}

\begin{abstract}
Relay technologies have been studied \& considered in the standardization process of the next generation of MIMO system, such as LTE 8, Advance LTE 10, IEEE 802.16e, IEEE 802.16m. Presently two wireless technologies, WiMax \& LTE both based on IEEE standard, are two rival technologies nevertheless, are technically very similar but deployment is differ. This introduces and compare features of two advance technologies in physical layer, and also gives performance analysis of different modulation schemes (BPSK, QPSK, and 16-QAM) in WiMAX \& LTE technologies.
\end{abstract}

Keywords: LTE 8, Advance LTE 10, IEEE 802.16e, IEEE 802.16m, MIMO system, WiMax.

\section{INTRODUCTION}

Mobile communications systems revolutionized the way people communicate, joining together communications and mobility. A long way in a remarkably short time has been achieved in the history of wireless. Evolution of wireless access technologies is about to reach its fourth generation (4G). Looking past, wireless access technologies have followed different evolutionary paths aimed at unified target: performance and efficiency in high mobile environment. The first generation $(1 \mathrm{G})$ has fulfilled the basic mobile voice, while the second generation (2G) has introduced capacity and coverage. This is followed by the third generation (3G), which has quest for data at higher speeds to open the gates for truly "mobile broadband" experience, which will be further realized by the fourth generation $(4 \mathrm{G})$.

The complexity of next generation wireless systems is growing exponentially. The combination of Multiple-Input Multiple-Output (MIMO) technology with Orthogonal Frequency Division Multiplexing (OFDM) is considered as the best solution to provide high data rates under frequency-selective fading channels.

The design and evaluation of MIMO-OFDM systems require a detailed analysis of a number of functionalities such as MIMO transmission modes, channel estimation, IMO detection, channel coding, across-layer scheduling . In this paper we present a MIMO OFDM-based simulator that includes the main physical and link layer functionalities. The (LTE) technology for different MIMO-OFDM techniques under realistic assumptions such as user mobility or bandwidth-limited feedback channel. Orthogonal Frequency Division Multiplexing (OFDM) is one of the most popular physical layer technologies for current broadband wireless communications due to its high spectral efficiency and robustness to frequency selective fading.
The use of Multiple -Output (MIMO) technology in combination with OFDM increases the diversity gain and/or the system capacity by exploiting spatial domain. Hence, MIMO-OFDM is an attractive solution for future broadband wireless systems like 3GPP Long-Term Evolution (LTE) and it will surely be a serious candidate for future $4 \mathrm{G}$ technologies. The evaluation of the physical layer performance under "realistic" varying channel conditions requires extremely time-consuming simulations. The use of specialized mathematical tools such as MATLAB/Simulink or Mathematica is widely extended to perform such simulations. These tools provide a wide set of built-in libraries that allow a rapid development of prototypes. There are other simulation tools like Visual System Simulator (VSS) or Ptolemy II that include standard building blocks to model and simulate complex communication systems. However, the performance evaluation of LTE technology requires specific MIMO-OFDM-based simulators to minimize the simulation time. An open-source MATLAB based LTE physical layer simulator is presented in nevertheless, it is proved that general purpose simulation platforms like MATLAB lead to very long execution times. Other works are only focused on the LTE simulation results under different MIMO-configurations, but no details on the employed simulator are provided. Besides, realistic assumptions such as user mobility, antenna correlation, or bandwidth limited feedback channel are often simplified or even omitted in some of previous works. This presents a new simulator for next generation MIMO-OFDM-based wireless systems.

The recent demand for higher data rate services from wireless network users is overwhelming. Social media influx as well as the proliferation of broadband enabled smart-phones, tablet computers and other newly improved wireless devices has erupted a new trend in wireless 
network traffic need where average capacity and speed is no longer appreciable. In order to cope with this trend in traffic requirement, wireless network operators are considering a gradual rollover of an existing third generation (3G) network to a fourth generation (4G) network with orthogonal frequency division multiple access (OFDMA) based technologies such as Fourth Generation Long Term Evolution (4G LTE) and Worldwide Interoperability for Microwave Access (WiMAX). This paper is devoted to the performance evaluation of Adaptive Modulation and Coding (AMC) in downlink of an orthogonal frequency division multiple access (OFDMA) network, considering Partial Usage of Sub-channels (PUSC). By using MATLAB Simulink and Origin 61, the performance of Bit Error Rate (BER) and Spectral Efficiency in two channel environments, i.e. nonfading and fading channels were examined. Results suggest better performance for AMC over individual MCS in all channel environments. Moreover, non-fading Addictive White Gaussian Noise (AWGN) channels significantly perform better than fading (Rayleigh and Rician) channels. In Rician channel environment, however, flat fading Rician channels perform better than frequency selective Rician channel which interestingly records a degraded performance against Rayleigh channels.

\subsection{Wi-Max}

Wi-Max used for high data rate over the large areas for a large number of users where broadband is not available. This is the first standard which can be used for fixed wireless access with effective higher bandwidth than the various cellular networks. Wireless broadband systems are basically used for many years but the main development of this standard enables economy of scale to bring down the cost of equipment, and decreases the envelop risk for operators. The 1st version of the IEEE 802.16 standard operates on $10-66 \mathrm{GHz}$ frequency band also requires line of sight (LOS) towers. But later the standard extended its operation of specification to $2-11 \mathrm{GHz}$ frequency band for non line of sight (NLOS). The aim of this paper is to transmit the data in WIMAX with low bit error rate and high efficient data with noisy area where we have to using Forward Error Correction method as Reed Solomon code and Convolution code. These methods are useful to decreases the BER and improve its Vefficiency. To reduce the noise these two error correction and error detection codes Reed Solomon coding and Convolution coding are used.

Now to increase the performance of the coding technique we are using Cyclic Prefix and interleaving techniques. So this paper is useful for analysis of WIMAX with different modulation techniques like BPSK, QPSK, QAM and also analysis of different efficiency of each modulation technique. According to the growth of multimedia services and the demand of Internet is to increasing interest in high speed communications. The requirement of wide bandwidth and flexibility improve the use of efficient transmission methods which fits the features of wideband channels basically in wireless environment in which the channels are very challenging. BWA is used due to its wireless nature, it is faster, easier to scale and more flexible. LTE (Long Term Evolution), a mobile telecommunication technology standardized by 3GPP, is the biggest jump on the evolution path from $3 \mathrm{G}$ UMTS and CDMA2000 towards 4G, with ambitious requirements for data rates, capacity and latencies. An advanced version of LTE, LTE-Advanced based on 3GPP UMTS Rel 10 in 2011, is also a 4G recognized mobile technology. The two technologies, Wi-Max and LTE, competed with each other starting their pre-4G versions and continued with their $4 \mathrm{G}$ versions while having much in common. It looks like that finally Wi-Max gave up the competition and selected to harmonize and integrate with LTE in its future harmonized Wi-Max advanced standard supporting multiple access technologies. This work addresses the technical similarities and differences between the two technologies trying to pinpoint those differences that advantage one technology over the other one. Other factors, commercial, historical, political, etc. which might advantage one technology over the other technology are also exploited. Finally, the article reviews plans of Wi-Max and LTE future with alternatives to Wi-Max discussed.

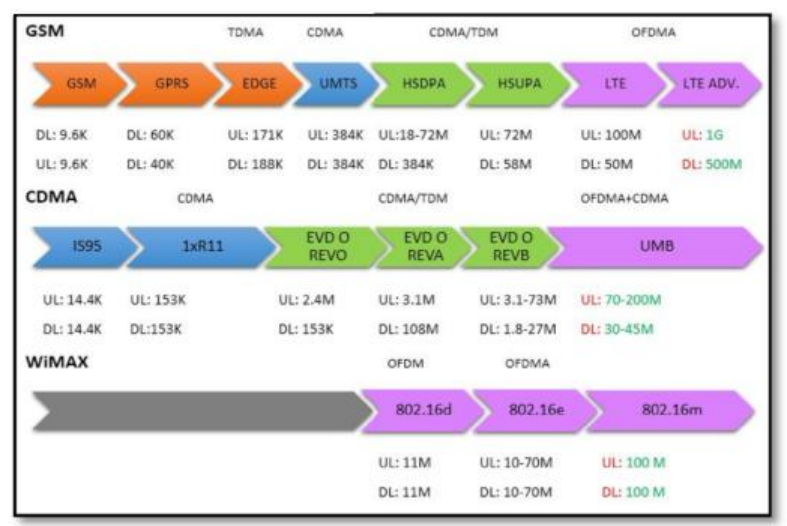

Fig1.1. Evolution of Advanced Technologies

\subsection{LTE}

The IEEE 802.16 group subsequently produced $802.16 \mathrm{a}$, an amendment to the standard, to include NLOS applications in the $2 \mathrm{GHz}-11 \mathrm{GHz}$ band, using an orthogonal frequency division multiplexing (OFDM)based physical layer. LTE is Wireless data communication standard was developed by 3GPP and was first commercialized by Telia Sonera in 2009 named as 4G LTE. LTE is the evolution of the GSM/UMTS standards. Goals of LTE was to increase the capacity, speed of wireless data networks using DSP technique, redesign and simplification of the network architecture, provide improved data rate, cell edge throughput, power consumption, latency, etc. The LTE wireless interface is incompatible with $2 \mathrm{G}$ and $3 \mathrm{G}$ networks; a separate wireless spectrum is required to implement LTE.

LTE provide lower data transfer latencies e.g. in optimal conditions with small IP packetslatency is sub-5 ms, LTE 
have lower latencies for handover and connection setup time then earlier radio access technologies.

Mobility Support: It has improved the mobility support for terminals with moving speed up to $350 \mathrm{~km} / \mathrm{h}$ or 500 $\mathrm{km} / \mathrm{h}$ depending upon frequency band.

Downlinks/Uplinks: To save power it uses OFDMA for downlinks and SC-FDMA for uplinks.

FDD and TDD support: LTE supports FDD, TDD, and half-duplex FDD with same radio technology.

Supporting frequency bands: LTE currently used by IMT systems by ITU-R, but it can support all frequency bands.

Spectrum flexibility: LTE increased standardized spectrum flexibility: $1.4 \mathrm{MHz}, 3 \mathrm{MHz}, 5 \mathrm{MHz}, 10 \mathrm{MHz}$, $15 \mathrm{MHz}$ and $20 \mathrm{MHz}$ wide cells.

Cell radius coverage support: LTE supports cell radius size from $10 \mathrm{~ms}$ up to $100 \mathrm{kms}$. For large distant rural areas with lower frequency bands, $5 \mathrm{~km}$ is optimal cell size; $30 \mathrm{~km}$ is reasonable performance, and $30-100 \mathrm{~km}$ cell size support acceptable performance. Higher frequency bands (e.g. $2.6 \mathrm{BHz}$ ) are supported into urban areas for high speed mobile broadband, but cell radius is limited to $1 \mathrm{~km}$ or less in this cases.Higher performance can be delivered with lower coverage area, but lower frequencies increase the coverage area and reduce the bandwidth.

\section{LITERATURE SURVEY}

\subsection{HISTORY}

2.1.1. First Generation (From now on, referenced as $1 \mathrm{G}$ ) $1 \mathrm{G}$ cellular networks were invented in the 1980s. The key idea behind $1 \mathrm{G}$ was that the geographical area is divided into cells (typically $10-25 \mathrm{~km}$ ), each served by a "base station." Cells are small so that frequency reuse can be exploited in nearby (but not adjacent) cells. This allows many more users to be supported in a given area. All 1G systems were analog systems popularly known as early cellular phone technology working in the frequency band of $150 \mathrm{MHz}$. The first commercially automated cellular network (the $1 \mathrm{G}$ generation) was launched in Japan by NTT (Nippon Telegraph and Telephone) in 1979, initially in the metropolitan area of Tokyo. Within five years, the NTT network had been expanded to cover the whole population of Japan and became the first nationwide $1 \mathrm{G}$ network.

\section{Technologies under 1G:}

$1 \mathrm{G}$ comprised of the following Mobile technologies: Mobile Telephone Systems (MTS), Advance Mobile Telephone Systems (AMTS), Push To Talk (PTT) and Improved Mobile Telephone Service (IMTS). Issues with 1G: Analog cellular phones are not very secure. Anyone with an all-band radio receiver connected to a computer can record the 32-bit serial numbers and phone numbers of subscribers when calling can listen in on any conversation. This loophole was exploited in many scandalous ways. There were also reported thefts of airtime. Anyone could collect a large database by driving around and go into business by reprogramming stolen phones and reselling them.

\subsection{SECOND GENERATION (From now on, referenced as $2 \mathrm{G})$}

$2 \mathrm{G}$ cellular telecom networks were commercially launched on the GSM standard in Finland by Radiolinja in 1991. 2G used digital signals for voice transmission and had a speed up to $64 \mathrm{kbps}$. It also provided the facility of Short Message Service (From now on, referenced as SMS) and used the bandwidth range of $30-200 \mathrm{KHz}$.

Technologies under 2G: $2 \mathrm{G}$ comprised of the following Mobile technologies:General Packet Radio Service (GPRS), CodeDivision Multiple Access (CDMA), Global Systemfor Mobile Communication (GSM) and EnhancedData Rates for GSM Evolution (EDGE).Some key benefits of $2 \mathrm{G}$ Network over itspredecessors was that, Digital Encryption wassupported by $2 \mathrm{G}$ systems which had higherpenetration efficiency thereby being more efficienton network spectrum. Moreover, 2G introducedseveral data services for mobile, the most prominentone being the famous SMS text messages. After 2 Gwas launched, the previous mobile telephonesystems were coined as $1 \mathrm{G}$ Systems. Although it hasbeen eons since its inception, $2 \mathrm{G}$ networks are stillused in many parts of the world.

Issues with $2 \mathrm{G}$ :In less populated areas, the weaker digital signaldeployed on higher frequencies may not be sufficientto reach a cell tower. Analog Signals have a smoothdecay curve while digital has a steppy one. This wasseen as both an advantage as well as a disadvantage.Under good conditions, digital sounded better. Under slightly bad conditions, analog experienced static, while digital has occasional dropouts. As conditions worsened, digital signals started to completely fail, while analog got worse gradually, generally holding a call longer and allowing at least a few words to get through. While digital calls tend to be free of static and background noise, the lossy compression used by the codecs takes a toll; the range of sound that is conveyed is reduced. You hear less of the tonality of someone's voice talking on a digital cell phone, but you will hear it more clearly.

\subsection{THIRD GENERATION (From now on, referenced} as 3G)

International Mobile Telecommunications-2000(IMT-2000), better known as $3 \mathrm{G}$ or 3rd Generation,is a generation of standards for mobile phones andmobile telecommunications services fulfilling theInternational Telecommunication Union. It usesWide Brand Wireless Network with which clarity isincreased. The data are sent through the technologycalled Packet Switching. Voice calls are interpretedthrough Circuit Switching. Along with verbalcommunication it includes data services, access totelevision/video, new services like Global Roaming.It operates at a range of $2100 \mathrm{MHz}$ and has abandwidth of $15-20 \mathrm{MHz}$ used for High-speedinternet service, video chatting. $3 \mathrm{G}$ uses Wide BandVoice Channel that is by this 
Vol. 6, Issue 1, January 2017

the world has beencontracted to a little village because a Technologies under 4G:

person cancontact with other person located in any part of $4 \mathrm{G}$ comprised of the following Mobile technologies: Long theworld and can even send messages too.
Technologies under 3G:

$2 \mathrm{G}$ comprised of the following Mobile technologies: $3 \mathrm{G}$ Technology comprises of Wideband CDMA, WLAN, Bluetooth, Universal Mobile telecommunication Systems (UMTS), eHigh Speed Downlink Packet Accss (HSDPA). Data are sent through packet switching. Voice calls are interpreted using circuit switching. It also provides facilities such as Global Roaming Clarity in voice calls, Fast Communication, Internet, Mobile T.V, Video Conferencing, Video Calls, Multi Media Messaging Service (MMS), 3D gaming and MultiplayerGaming.

\section{Issues with 3G:}

Although the usage price of $3 \mathrm{G}$ technology has greatly reduced since its inception due to wider adoption, it still is very costly as compared to $2 \mathrm{G}$ technologies. Due to high bandwidth transmission of 3G technologies, power consumption greatly increases which results in reduced device battery life. The data consumption of $3 \mathrm{G}$ at times becomes so heavy due to the high transmission rates that it puts a big load on the network; to alleviate which, many cellular operators implemented data usage caps which were disadvantageous to customers.
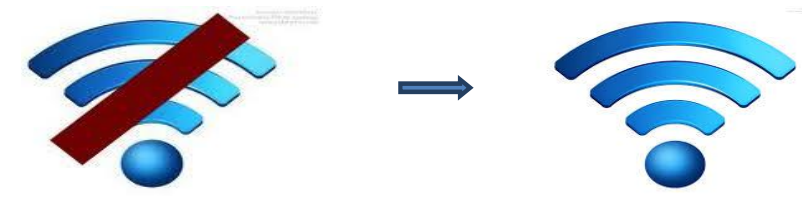

Fig 2.1 Mission of Project

- Slow internet to fast.

- Sometimes even after having the internet the speed is a big issue.

- For this our aim is to bring the high speed internet.
Term Evolution (LTE) Standard based on the GSM/EDGE and UMTS/HSPA, 3rd Generation Partnership Project (3GPP), Multiple In Multiple Output (MIMO) smart antenna technology, Orthogonal Frequency Digital Multiplexing (OFDM), 802.16e - Worldwide Interoperability for Microwave Access (WiMAX), 802.20 - Mobile Broadband Wireless Access (MBWA)

\section{Issues with 4G:}

$3 \mathrm{G}$ and $4 \mathrm{G}$ components made for one continent is not always compatible with another continent sue to carrying frequency bands. Another prominent issue in $4 \mathrm{G}$ systems is to make higher bit rates available in larger portion of the cell, especially to users in an exposed position in between several base stations. In current research, this issue is addressed by macrodiversity techniques, also known as group cooperative relay, and also by Beam-Division Multiple Access (BDMA). Pervasive networks are a hypothetical amorphous concept where the user can be simultaneously connected to several wireless access technologies and can seamlessly move between them. This technology has not yet been efficiently implemented.

\subsection{FIFTH GENERATION (From now on, referenced} as 5G)

The evolution of LTE does not end with LTE advanced (release 10) rather continues to evolve into further releases. Each new release further enhances system performance and adds new capabilities with new application areas. Some of the additional applications, benefiting from mobile connectivity, are home automation, smart transportation, security, etc.

\section{Need for 5G:}

From user point of view, apart from throughput,other factors that differentiate $5 \mathrm{G}$ from itspredecessors and makes its implementation essentialare:

\subsection{FOURTH GENERATION (From now on, referenced as 4G)}

In March 2008, the International Telecommunications Union-Radio communications sector (ITU-R) specified a set of requirements for $4 \mathrm{G}$ standards, named the International Mobile Telecommunications Advanced (IMT-Advanced) specification, setting peak speed requirements for $4 \mathrm{G}$ service at 100 megabits per second (Mbit/s) for high mobility communication (such as from trains and cars) and 1 gigabit per second (Gbit/s) for low mobility communication (such as pedestrians and stationary users). A 4G system not only provides voice and other $3 \mathrm{G}$ services but also provides ultra broad band network access to mobile devices. Applications vary from IP telephony, HD Mobile Television, video conferencing to gaming services and cloud computing. One of the initial devices to access $4 \mathrm{G}$ network was USB wireless modem which was later followed by cellular phones with WiMax and LTE technology. 
- 5G technology offer high resolution for crazy cell phone user and bi-directional large bandwidth shaping .

- 5G technology offer transporter class gateway with unparalleled consistency . 5G Network Model is an All-IP based model for mobile and wireless network interoperability. The All-IP Network (from now on referenced as AIPN) has the capacity to satisfy the ever increasing mammoth demands of the burgeoning cellular market. It also is a general platform for all radio access technologies and standards. All-IP Network uses packet switching as compared to circuit switching used its predecessors, and its continual evolution provides performance and cost optimization. In 5G, Network Architecture consists of a user terminal (which has a crucial role in the new architecture) and a number of independent, autonomous radio access technologies (from now on referenced as RAT) .AIPN based mobile applications and services such as Mobile Portals, Mobile Commerce, Mobile Health-Care, Mobile Government, Mobile Banking and several others are offered via Cloud Computing Resources (from now on referenced as CCR). The best feature about cloud computing is that a user can access any data uploaded on the cloud ubiquitously from anywhere, from any terminal with an internet connection or a secure connection to the storage cloud without the need to install any third party application or softwares.

\section{DEVELOPMENT OF SYSTEM}

\subsection{SIMULATION AND RESULT}

Simulation procedure

\subsubsection{Run Transmitter}

The first step in the simulation is to use the transmitter to create a digitally modulated signal from a sequence of pseudo-random bits. Once we have created this signal, $\mathrm{x}(\mathrm{n})$,we need to make some measurement so fit.

\subsubsection{Establish SNR}

The signal-to-noise-ratio(SNR),Eb/N0, is usually expressed in decibels, but we must convert decibels to an ordinary ratio before we can make further use of the SNR. If We set the $\mathrm{SNR}$ to $\mathrm{m} \mathrm{dB}$, then $\mathrm{Eb} / \mathrm{N} 0=10 \mathrm{~m} / 10$.

Using Matlab, we find the ratio, 'ebn0',from the SNR in decibels, 'snrdb', as: ebn $0=10^{\wedge}($ snrdb/10). Note that $\mathrm{Eb} / \mathrm{NO}$ is a dimensionless quantity.

\subsubsection{Determine Eb}

Energy-per-bitis the total energy of the signal, divided by the number of bits contained in the signal. We can also express energy-per-bit as the average signal power Multiplied by the duration of one bit. Either way,the expression for $\mathrm{Eb}$ is: $\mathrm{Eb}=1 \mathrm{~N} \cdot \mathrm{f}$ bit $\mathrm{N} \mathrm{n}=1 \times 2(\mathrm{n})$, Where $\mathrm{N}$ is the total number of samples in the signal, and $\mathrm{fbit}$ is the bit rate in bits per-second. Using Matlab, we find the energy-per-bit,'eb', of our transmitted signal, ' $x$ ', that Has abit rate' $\mathrm{fb}$ ',as: eb $=\operatorname{sum}\left(\mathrm{x} .{ }^{\wedge} 2\right) /($ length $(\mathrm{x}) * \mathrm{fb})$. Since our signal, $\mathrm{x}(\mathrm{n})$, is in units of volts, the units of $\mathrm{Eb}$ are Joules.

\subsubsection{CALCULATE No}

With the SNR and energy-per bit now known,we are ready to calculateN0,the one sided power spectral density of the noise. All we have to do is divide Eb by the SNR, Providing we have converted the SNR from decibels to a ratio. Using Matlab, we find the power spectral density of

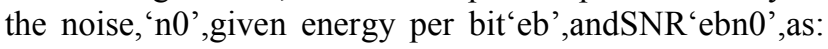
$\mathrm{n} 0=\mathrm{eb} / \mathrm{ebn} 0$. The power spectral density of the noise has units of Watts per Hertz.

\section{PROGRAM CODE:}

clear

$\mathrm{N}=10^{\wedge} 6$

rand('state',100);

randn('state',200);

$\%$ Transmitter

$\mathrm{ip}=\operatorname{rand}(1, \mathrm{~N})>0.5$;

$\mathrm{s}=2 * \mathrm{ip}-1$

$\mathrm{n}=1 / \operatorname{sqrt}(2) *[\operatorname{randn}(1, \mathrm{~N})+\mathrm{j} * \operatorname{randn}(1, \mathrm{~N})]$;

Eb_NO_dB $=[-3: 10]$;

for ii $=1$ :length(Eb_N0_dB)

$\%$ Noise addition

$\mathrm{y}=\mathrm{s}+10^{\wedge}\left(-\mathrm{Eb} \_\mathrm{NO} \_\mathrm{dB}(\mathrm{ii}) / 20\right) * \mathrm{n}$

$\%$ receiver - hard decision decoding

ipHat $=\operatorname{real}(y)>0$;

$\%$ counting the errors $\mathrm{nErr}(\mathrm{ii})=\operatorname{size}($ find $([\mathrm{ip}-\mathrm{ipHat}]), 2)$;

end

simBer $=\mathrm{nErr} / \mathrm{N}$;

theoryBer $=0.5 * \operatorname{erfc}\left(\operatorname{sqrt}\left(10 .^{\wedge}\left(E b \_N 0 \_d B / 10\right)\right)\right)$;

$\%$ plot

close all

figure

semilogy(Eb_N0_dB,theoryBer,'b.-');

hold on

semilogy(Eb_N0_dB,simBer,'mx-');

$\operatorname{axis}\left(\left[\begin{array}{llll}-3 & 10 & 10^{\wedge}-5 & 0.5\end{array}\right]\right)$

grid on

legend('theory', 'simulation');

xlabel('Eb/No, dB');

ylabel('Bit Error Rate');

title('Bit error probability curve for BPSK modulation');

\subsection{Simulation Result}

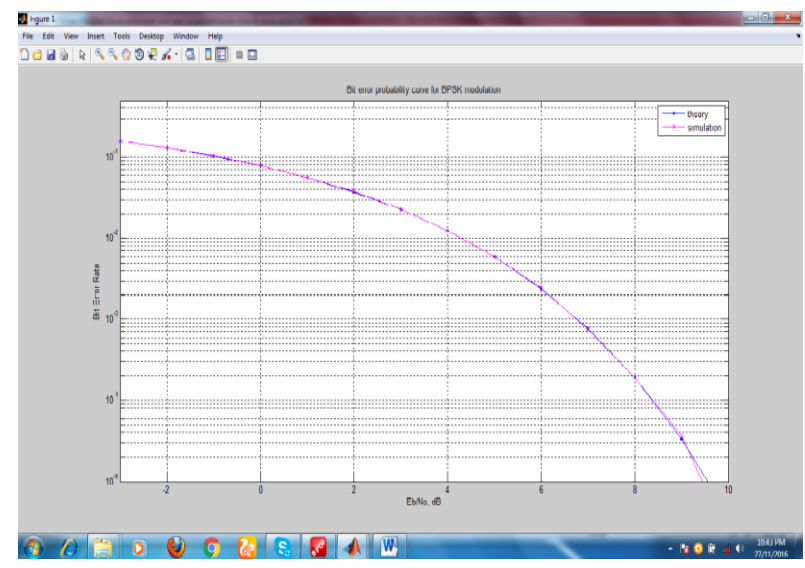




\section{REFERENCES}

[1] R. Pazhyannur, "Comparison of LTE and WiMAX," in IP NGN Architecture Leadership Journal Q, 2010.

[2] Yang Yang, Honglin $\mathrm{Hu}$, Jing $\mathrm{Xu}$, and Guoquiang Mao, "Relay Technologies for WiMAX and LIE-Advanced Mobile Systems.," IEEE Commun. Mag., vol. 47, no. October, pp. 100-105, 2009.

[3] D. M. K.N.S. Kumar, M.Kata, P.Chaitanya, "LTEAdvanced鋛:Future of Mobile Broadband," 2009.

[4] "The Mobile Broadband Standard," Global Initiative. [Online]. Available: www.3gpp.org. [Accessed: 20-Jan-2015].

[5] "Performance analysis of both WIMAX and LTE Master MDM Internship Performance analysis of both WIMAX and LTE technologies Supervisor 銈: Salima Hamma,” 2013.

[6] H. Ekström, A. Furuskär, J. Karlsson, M. Meyer, S. Parkvall, J. Torsner, and M. Wahlqvist, "Technical solutions for the $3 \mathrm{G}$ longterm evolution," IEEE Commun. Mag., vol. 44, no. March, pp. $38-45,2006$. 